\title{
Tristability in Cancer-Associated MicroRNA-TF Chimera Toggle Switch
}

Mingyang Lu, ${ }^{* \dagger}$ Mohit Kumar Jolly, ${ }^{\dagger, \dagger}$ Ryan Gomoto, ${ }^{\dagger, \#}$ Bin Huang, ${ }^{\dagger, \S}$ José Onuchic, ${ }^{\dagger, \S, \|, \perp}$ and Eshel Ben-Jacob* $* \dagger, \perp, \Phi$

${ }^{\dagger}$ Center for Theoretical Biological Physics, ${ }^{\ddagger}$ Department of Bioengineering, ${ }^{\S}$ Department of Chemistry, "Department of Physics and Astronomy, ${ }^{\perp}$ Department of Biochemistry and Cell Biology, Rice University, Houston, Texas 77005-1827, United States

${ }^{\#}$ Graduate Program in Structural and Computational Biology and Molecular Biophysics, Baylor College of Medicine, Houston, Texas 77030, United States

${ }^{\text {II }}$ School of Physics and Astronomy and The Sagol School of Neuroscience, Tel-Aviv University, Tel-Aviv 69978, Israel

Supporting Information

ABSTRACT: Cell fate decisions during embryonic development and tumorigenesis pose a major research challenge in modern developmental and cancer biology. Binary cell fate decisions are usually regulated by gene circuits incorporating either classical toggle switches with two mutually inhibiting transcription factor (TF) genes or chimera toggle switches with a mutually inhibiting pair of microRNA (miRNA) and TF gene. These circuits can explain binary cell fate decisions. Importantly, intermediate cell types can exist during the differentiation of both stem cells and cancer cells. It has been shown that TF-TF self-activating toggle switches (SATS) can have coexistence of three metastable states (tristability), yet the role of chimera toggle switches in opening these additional states remains elusive. Here we present a generalized framework for both the TF-TF SATS and miRNA-TF chimera SATS, starting from the TF-promoter and miRNA-mRNA binding/unbinding dynamics. We show that the chimera SATSs can also have tristability. We demonstrate that the dynamics of miRNA-TF SATS is qualitatively different from that of the TF-TF SATS because the nonlinear effects of translational silencing by miRNA are distinct from those of transcriptional repression. We discuss the possible relevance of these findings to fate decisions by cancer cells.

\section{INTRODUCTION}

Over recent years, we have witnessed rapid progress in mapping the gene regulatory networks associated with cellular differentiation, programmability, and reprogramming. We refer to differentiation of pluri- and multipotent stem and progenitor cells during embryonic development and tissue regeneration, to differentiation and programmability of cancer and cancer stemlike cells during tumorigenesis, and to the reprogramming of differentiated cells during generation of induced pluripotent stem cells (iPSCs). Cell fate determinations in all of these examples involve changes in expression of various transcription factors (TFs) and microRNAs (miRNAs) that regulate cascades of regulatory networks, ultimately generating genome-wide gene expression patterns corresponding to a particular cell lineage (fate).

The decision networks involved in the aforementioned cell fate determinations have a convoluted architecture that varies from example to example, ${ }^{1,2}$ yet the elaborated complexity of these signal transduction networks can be simplified. Similar to bacterial decision networks, ${ }^{3}$ the eukaryote decision networks' organization is that of linked simpler modules, each with its own functional role. As it turns out, the description and modeling of the dynamics of several of the key modules can further be simplified as they are composed of either interacting twocomponent circuits (mainly toggle-switch-like) or two-component circuits coupled to three-component circuits (mainly feedforward-loop-like).

The core two-component circuits in many decision modules have the architecture of the classical TF-TF toggle switch, a regulatory motif in which the proteins of two genes act as mutually inhibiting TFs. Typically, one or both genes also act as self-activating TFs (directly or through other genes), thus forming a "self-activating toggle switch" (SATS). We refer to the case when both genes are self-activating as a symmetric SATS and to the case when only one gene is self-activating as an asymmetric SATS.

Two well-studied examples are: the (PU1-GATA1) pair, ${ }^{4}$ which governs the differentiation of a common myeloid progenitor into two different hematopoietic lineages, and the (CDX2-OCT3/4) pair, ${ }^{5}$ which is associated with the differentiation of embryonic stem (ES) cells.

Special Issue: Peter G. Wolynes Festschrift

Received: March 30, 2013

Published: May 16, 2013 
The realization that self-activating TF-TF toggle switches play an important role during embryonic development led several groups to carry theoretical studies of such circuits. Most took the dynamical system approach to inspect the possible states and their relative stability as a function of the circuit architecture and parameters. These studies revealed that the SATS elements can yield for some range of parameters the coexistence of three metastable states (tristability). ${ }^{6-8}$ Although each of the groups described the SATS by different deterministic equations, they reached similar conclusions.

Here we present a generalized framework for modeling SATSs starting with the basic process of $\mathrm{TF}$ binding/unbinding dynamics. ${ }^{9-11}$ In the main text, we consider the noncompetitive case in which the promoter has two binding sites (one for each $\mathrm{TF}$ ). In the Supporting Information (SI), we analyze the competitive case in which the promoter has a single binding site for both TFs. We also extended the previous studies by including an external input signal I that acts as a TF, and thus it mimics driving the SATS by another gene circuit.

The TF-TF circuits are only part of the picture: it is wellestablished that translational regulation by miRNA plays a crucial role during embryonic development and tumorigenesis ${ }^{12,13}$ and that many of the decision modules encompass miRNAs. ${ }^{14}$ In particular, "translation-transcription chimera" toggle switches comprising miRNA-TF pairs were found to play an important role in fate determination by cancer cells. For instance, the (Lin28-let-7) pair forms such a switch that is associated with regulation of self-renewal of special programmable dormant cancer cells (PDCCs) commonly referred to as cancer stem cells. ${ }^{15}$ The well-studied miR-200, miR-128-2, and miR-34 form chimera switches with SNAIL, ZEB1/2, and GATA3 - TFs that regulate epithelial-to-mesenchymal transition (EMT) ${ }^{16-20}$ and the reverse transitions (MET). Later, we show examples of selfactivating TF-TF and miRNA-TF toggle switches (Figure 1) and how these simple circuits are embedded in more elaborated decision modules (Figure 2) in the context of both ES cells and cancer cells. $^{19,21-25}$

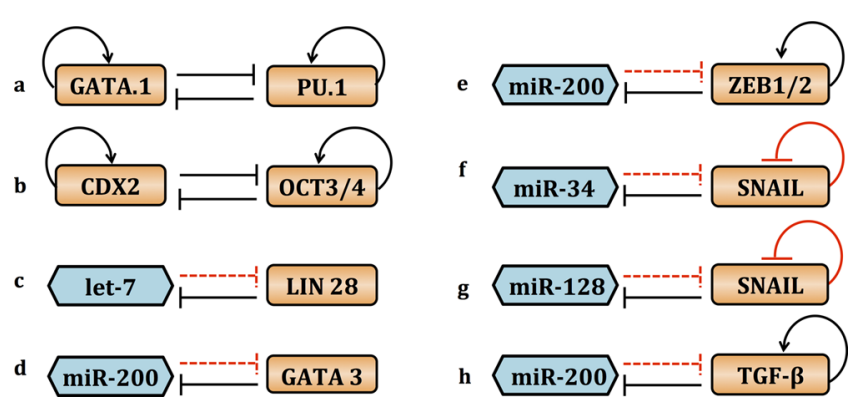

Figure 1. Examples of toggle switches like decision circuits. Brown rectangular boxes are for transcription factors, whereas blue hexagonal boxes denote microRNAs. Solid black line denotes transcriptional repression; red dotted line denotes repression through microRNA. Red and black circular arrows denote negative and positive autoregulation, respectively.

To incorporate the essential feature of miRNA-mediated regulation, we generalized the theoretical framework for modeling self-activating miRNA-TF chimera toggle switches. We devised a model for the miRNA-TF chimera switches by starting from the TF-promoter binding/unbinding dynamics and adding the miRNA-mRNA binding/unbinding dynamics. We note that the three-coupled deterministic equations, derived by
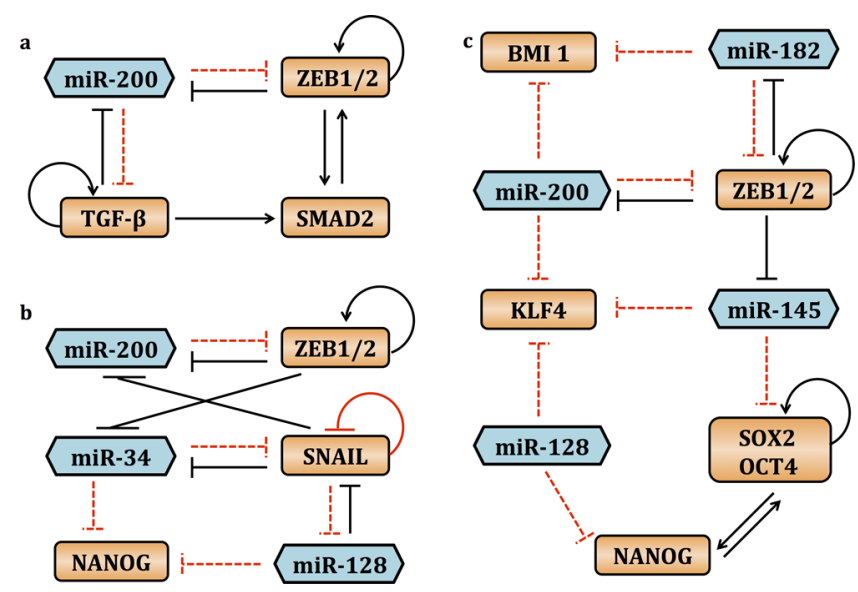

Figure 2. Examples of decision modules that contain the toggle-switchlike circuits. The module (a) contains two asymmetric SATS circuits; the module (b) contains one asymmetric SATS circuit and two toggle switches with one-sided self-inhibition; and the module (c) contains two asymmetric SATS circuits.

this more biologically consistent approach, qualitatively differ from the deterministic equations studied in the context of miRNA-TF toggle switch without self-activation. ${ }^{26,27}$ We also note that the equations incorporate (in addition to the Hill functions) a new type of functions $\mathrm{M}^{+}(\mu)$ and $\mathrm{M}^{-}(\mu)$ (where $(\mu)$ is the level of miRNA), which play the role of the inhibitory and excitatory Hill function when miRNAs are involved.

Dynamical system analyses of the new model revealed that the chimera SATSs can yield both bistability and tristability similar to the TF SATS, for a significant range of biologically reasonable circuit parameters (SI). In the Discussion section, we reflect on the implication of these findings with regards to the cell fate determination of cancer cells and the possible association between differentiation and stemness of cancer cells.

Examples of Two Component Decision Circuits. In this section, we present some examples of miRNA-TF chimera toggle switches as well as self-activating TF-TF switches. PU.1 and GATA-1 (Figure 1a) are lineage-specific TFs that regulate the differentiation of a common myeloid progenitor into erythrocyte and macrophage. ${ }^{4}$ They are mutually antagonistic and promote their own production, thus forming an SATS. ${ }^{28}$ Similarly, CDX2 and OCT3/4 (Figure 1b) govern the differentiation of an ES cell by reciprocal inhibition of their target genes. ${ }^{5}$ An important example of an miRNA-TF chimera toggle switches is the (Lin28-let-7) (Figure 1c) pair controlling the self-renewal of mammary epithelial progenitor cells and cancer cells. ${ }^{15}$ Many such chimera switches are found in the context of EMT, a dedifferentiation program through which cancer cells lose their epithelial phenotype and gain a mesenchymal migratory one to initiate metastasis. ${ }^{29,30}$ miR-200 is a powerful inducer of epithelial differentiation and suppresses EMT, whereas ZEB1/ 2 is a TF that activates EMT (EMT-TF) and is overexpressed in cancer cells with mesenchymal morphology. ZEB1/2 directly inhibits the transcription of miR-200 genes and is directly targeted by miR-200. ${ }^{16,20}$ Furthermore, we hypothesize that the ZEB1/2 activates itself directly or indirectly. ${ }^{31}$ This selfactivating chimera switch of ZEB1/2 and miR-200 regulates EMT (Figures 1e and 2a). The miRNA miR-200 also forms toggle switches with other potent EMT inducers (Figure 1d,f), such as TGF- $\beta$ and GATA3. ${ }^{18,24}$ Similarly, another EMT-TF, SNAIL, represses itself and forms chimera switches with miR-34 
(a)

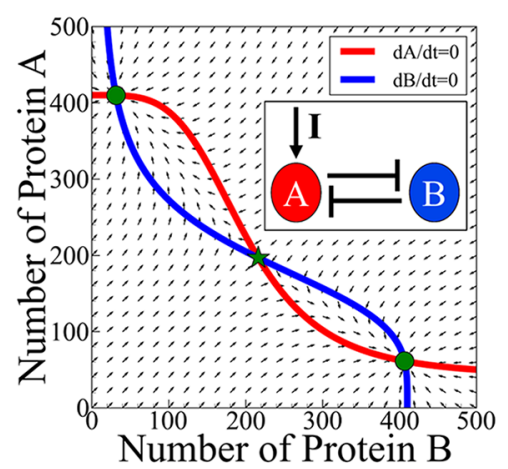

(c)

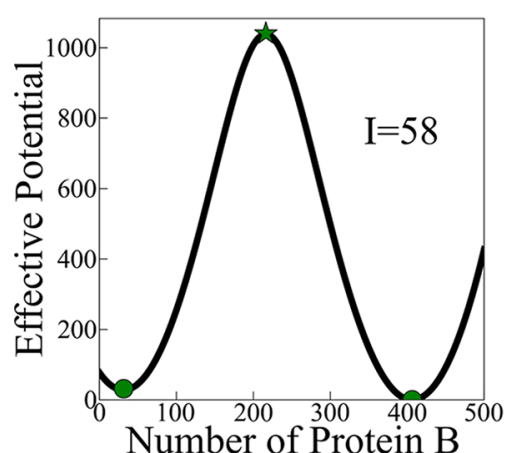

(b)

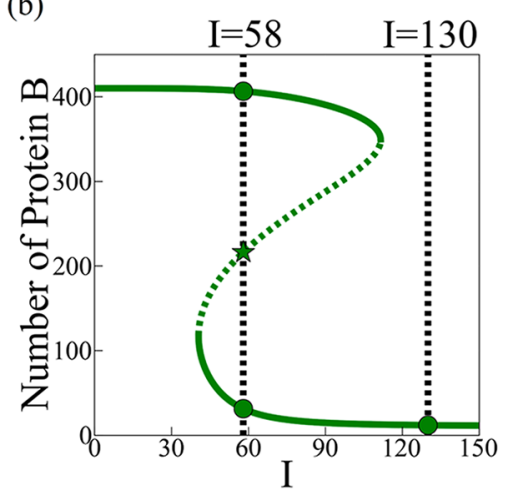

(d)

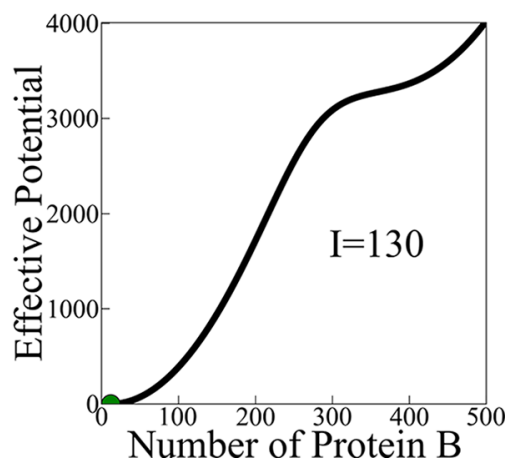

Figure 3. Classical toggle switch driven by an external signal. The results shown in this Figure were computed for the schematic circuit depicted in panel A from eq 1. (a) Phase space showing the nullclines for a specific value of the input signal I when two metastable states coexist. (b) Bifurcation diagram as a function of the input signal. The green solid lines represent the stable fixed points, while the green dotted line represents the unstable fixed points. The two specified cases: two coexisting metastable states (bistability on the left, $\mathrm{I}=38$ ) and single metastable states (the unistability on the right, $\mathrm{I}=80$ ). Panels $\mathrm{c}$ and $\mathrm{d}$ show a 1-D effective potential computed along the $\mathrm{dA} / \mathrm{d} t=0$ nullcline for $\mathrm{I}=38$ and 80 , respectively. Green circles mark the stable fixed points, and the green star marks the unstable saddle fixed point.

and miR-128-2 (Figure 1g,h), another set of gatekeepers of the epithelial phenotype. ${ }^{17,19,32}$ The miRNAs miR-200, miR-34, and miR-128-2 (Figure 2b,c) repress regulators of self-renewal of embryonic and cancer stem cells such as KLF4, BMI1, and NANOG, ${ }^{22,33}$ thus indicating some links between EMT and stemness.

\section{RESULTS}

First, we introduce a generalized theoretical framework for the transcriptional regulation by two TFs. We then implement this method to model the asymmetric TF SATS and the symmetric TF SATS. Next, we devise a new generalized model for both the transcriptional regulation and translational regulation by a, miRNA and a TF and implement this new method to model the miRNA-TF chimera SATS.

Classical TF Toggle Switch. The classical toggle switch can have two stable states $(0,1)$ and $(1,0)$, where state $(0)$ corresponds to relatively low expression (low concentration) and state (1) corresponds to relatively high expression. The two stable states, which correspond to two stable fixed points in the phase space, are separated by an unstable saddle fixed point. (See Figure 3.) Unlike previous studies, we hereby present a bifurcation diagram as a function of a driving signal stimulating the transcription of one of the genes to mimic the situation that the toggle switch is coupled to another circuit. Therefore, the input signal is represented as an additional TF I. The resulting deterministic equations for the noncompetitive case in which the promoter of gene A has two binding sites, one for I and one for B, are:

$$
\begin{aligned}
& \mathrm{dA} / \mathrm{d} t=G_{\mathrm{A}}(\mathrm{I}, \mathrm{B})-k_{\mathrm{A}} \mathrm{A} \\
& \mathrm{dB} / \mathrm{d} t=g_{\mathrm{B}, 0}+g_{\mathrm{B}, \mathrm{A}} \mathrm{H}^{-}(\mathrm{A})-k_{\mathrm{B}} \mathrm{B}
\end{aligned}
$$

The derivation of the equations and the definition of $G_{\mathrm{A}}(\mathrm{I}, \mathrm{B})$ are presented in the next section.

The circuit parameters for all cases are presented in the SI. The level of the TF, mRNA, and miRNA are measured in number of molecules and time is measured in minutes. We use the following definitions for inhibitory and excitatory one-TF Hill functions:

$$
\begin{aligned}
& \mathrm{H}^{-}(\mathrm{A}) \equiv 1 /\left[1+\left(A / A_{0}\right)^{n_{\mathrm{A}}}\right] \\
& \mathrm{H}^{+}(\mathrm{A}) \equiv\left(A / A_{0}\right)^{n_{\mathrm{A}}} /\left[1+\left(A / A_{0}\right)^{n_{\mathrm{A}}}\right]=1-\mathrm{H}^{-}(\mathrm{A})
\end{aligned}
$$

The resulting deterministic equations for the competitive case (one binding site) are:

$$
\begin{aligned}
\mathrm{dA} / \mathrm{d} t= & g_{\mathrm{A}, 0} \mathrm{H}^{-}(\mathrm{I}, \mathrm{B})+g_{\mathrm{A}, \mathrm{I}} \mathrm{H}^{+}(\mathrm{I}, \mathrm{B})+g_{\mathrm{A}, \mathrm{B}} \mathrm{H}^{+}(\mathrm{B}, \mathrm{I}) \\
& -k_{\mathrm{A}} \mathrm{A} \\
\mathrm{dB} / \mathrm{d} t= & g_{\mathrm{B}, 0}+g_{\mathrm{B}, \mathrm{A}} \mathrm{H}^{-}(\mathrm{A})-k_{\mathrm{B}} B
\end{aligned}
$$

where the generalized two-TF Hill functions $\mathrm{H}^{-}(\mathrm{A}, \mathrm{B})$ and $\mathrm{H}^{+}(\mathrm{A}, \mathrm{B})$ are defined as,

$$
\begin{aligned}
& \mathrm{H}^{-}(\mathrm{A}, \mathrm{B}) \equiv 1 /\left[1+\left(A / A_{0}\right)^{n_{\mathrm{A}}}+\left(B / B_{0}\right)^{n_{\mathrm{B}}}\right] \\
& \mathrm{H}^{+}(\mathrm{A}, \mathrm{B}) \equiv\left(A / A_{0}\right)^{n_{\mathrm{A}}} /\left[1+\left(A / A_{0}\right)^{n_{\mathrm{A}}}+\left(B / B_{0}\right)^{n_{\mathrm{B}}}\right]
\end{aligned}
$$




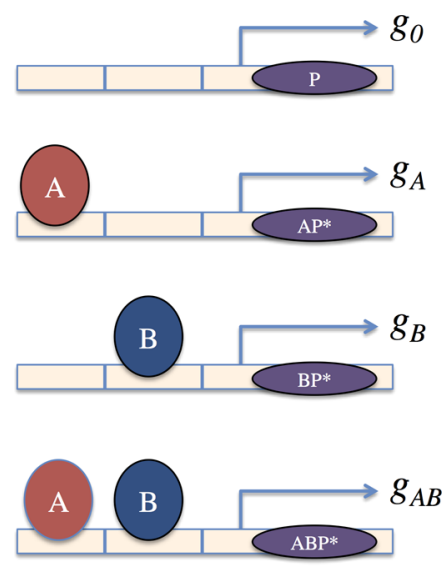

$-A-$

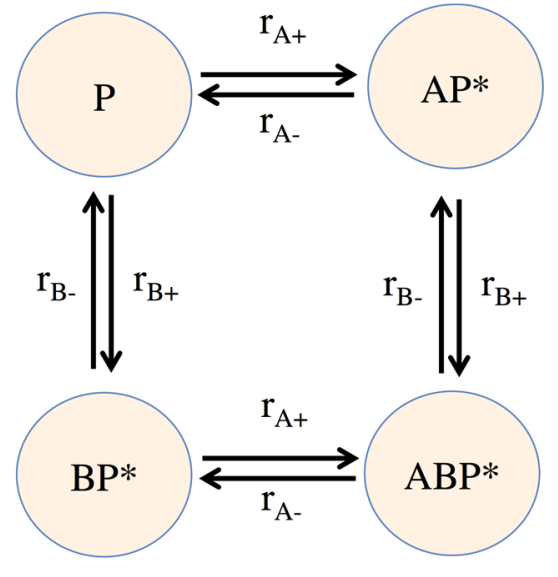

-B-

Figure 4. Illustration of the promoter-TFs binding-unbinding dynamics for the case of two binding sites. (A) Illustration of the four states of the promoter, as is described in the text. The transcription rates for each promoter state are denoted by $g_{\mathrm{X}, 0}, g_{\mathrm{X}, \mathrm{A}}, g_{\mathrm{X}, \mathrm{B}}$, and $g_{\mathrm{X}, \mathrm{AB}}$, corresponding to $\mathrm{P}, \mathrm{AP} *$, $\mathrm{AP}^{*}$, and $\mathrm{ABP} *$, respectively. Note that in panel A we omit the $\mathrm{X}$ (e.g., we use $g_{0}$ instead of $g_{\mathrm{X}, 0}$ ) to simplify the Figure. (B) Chemical reactions of the binding and unbinding of the TFs involved in the transitions between the four states of the promoter.

The phase space for the noncompetitive case for a specific value of the input signal I is illustrated in Figure 3a, and the bifurcation diagram as a function of the input signal $I$ is presented in Figure 3b. In Figure 3c,d we show two examples (for two different values of the input signal) of the 1-D potential energy obtained by integrating the equations along the nullcline $\mathrm{dA} / \mathrm{d} t=0$. Analysis of the corresponding competitive case is presented in the SI.

Generalized Framework of Gene Regulation by Two Transcription Factors. Many genes participating in twocomponent circuits are regulated by two TFs. Therefore, it is essential to develop a generalized modeling framework for consistent description of gene regulation by two TFs starting from the promoter-TF binding/unbinding dynamics. Typically, the promoter has two binding sites, one for each TF. In this section, we model this noncompetitive case. The less common competitive case in which the two TFs compete in binding to a single common binding site is discussed in the SI.

As is illustrated in Figure 4A, for the two binding sites case, the promoter can be in one of four states: free form denoted by $\mathrm{P}$, one-TF-bounded form (either A or B are bounded) denoted by $\mathrm{AP}^{*}$ and $\mathrm{BP} *$, respectively, and two-TF-bounded form denoted by $A B P^{*}$. The transitions among these states involve binding and unbinding of the TF A or B, as is illustrated in Figure 4B. We note that in this noncompetitive case the coupling between the two TFs resulted from the fact that the probabilities of the four states have to add to one, that is:

$$
[\mathrm{P}]+\left[\mathrm{AP}^{*}\right]+\left[\mathrm{BP}^{*}\right]+[\mathrm{ABP} *]=1
$$

Using eq 5 and the scheme of transition rates between the four promoter states (Figure 4B), it can be shown (see SI) that the probabilities of the four promoter states are given by:

$$
\begin{aligned}
& {[\mathrm{P}]=\mathrm{H}^{-}(\mathrm{A}) \mathrm{H}^{-}(\mathrm{B})} \\
& {\left[\mathrm{AP}^{*}\right]=\mathrm{H}^{+}(\mathrm{A}) \mathrm{H}^{-}(\mathrm{B})} \\
& {\left[\mathrm{BP}^{*}\right]=\mathrm{H}^{-}(\mathrm{A}) \mathrm{H}^{+}(\mathrm{B})} \\
& {\left[\mathrm{ABP}^{*}\right]=\mathrm{H}^{+}(\mathrm{A}) \mathrm{H}^{+}(\mathrm{B})}
\end{aligned}
$$

Using these probabilities combined with eq 5 and the definitions of the transcription rates of the four promoter states (Figure 4A), we obtain that the total mean transcription rate $G_{X}(A, B)$ of a gene $\mathrm{X}$ that is regulated by two TFs $\mathrm{A}$ and $\mathrm{B}$ in the case of two binding sites is given by:

$$
\begin{aligned}
G_{\mathrm{X}}(\mathrm{A}, \mathrm{B})= & \left(g_{\mathrm{X}, 0}-g_{\mathrm{X}, \mathrm{A}}-g_{\mathrm{X}, \mathrm{B}}+g_{\mathrm{X}, \mathrm{AB}}\right) \mathrm{H}^{-}(\mathrm{A}) \mathrm{H}^{-}(\mathrm{B}) \\
& +\left(g_{\mathrm{X}, \mathrm{B}}-g_{\mathrm{X}, \mathrm{AB}}\right) \mathrm{H}^{-}(\mathrm{A})+\left(g_{\mathrm{X}, \mathrm{A}}-g_{\mathrm{X}, \mathrm{AB}}\right) \\
& \mathrm{H}^{-}(\mathrm{B})+g_{\mathrm{X}, \mathrm{AB}}
\end{aligned}
$$

Note that this is a general result: each of the two TFs A and B can be either inhibitory $\left(g_{\mathrm{A}}\right.$ and $g_{\mathrm{B}}$ are no larger than $\left.g_{0}\right)$ or excitatory $\left(g_{\mathrm{A}}\right.$ and $g_{\mathrm{B}}$ are no smaller than $\left.g_{0}\right)$. Equation 7 can also be applied to the situations in which the transcription rate is altered (increased or decreased) for some specific promoter state(s). For example, in the situation of a restrictive AND logic on two activating TFs, the transcription rate is only enhanced for the $\mathrm{ABP}^{*}$ state. See the SI for the detailed analyses on some special cases. We also note that in general, gene $\mathrm{X}$ can be one of the genes $\mathrm{A}$ or $\mathrm{B}$. In the case of input signal I that acts as a TF, either A or B can be replaced by I, as we show later.

When the transcription rates satisfy the following relations: $g_{\mathrm{X}, \mathrm{A}} \gg g_{\mathrm{X}, 0}, g_{\mathrm{X}, \mathrm{B}} \ll g_{\mathrm{X}, 0}$, and $g_{\mathrm{X}, \mathrm{AB}} \approx g_{\mathrm{X}, 0}, G_{\mathrm{X}}(\mathrm{A}, \mathrm{B})$ can be approximated by

$$
\begin{aligned}
G_{\mathrm{X}}(\mathrm{A}, \mathrm{B}) \approx & {\left[g_{\mathrm{X}, \mathrm{AB}}+g_{\mathrm{X}, \mathrm{A}} \mathrm{H}^{-}(\mathrm{B})\right] \mathrm{H}^{+}(\mathrm{A})+g_{\mathrm{X}, \mathrm{B}} } \\
& +g_{\mathrm{X}, 0} \mathrm{H}^{-}(\mathrm{B})
\end{aligned}
$$

While the eq 8 is a good approximation (see the SI for derivation), the results presented later were obtained using the full expression in eq 7 .

Deterministic Model of the Self-Activating Toggle Switch. Following the generalized theoretical framework devised above, we analyze the dynamics of the SATS circuits for the case of two binding sites. Figure 5A shows schematics and the transcription rates for the asymmetric SATS (only gene B is self-activating), and Figure $5 \mathrm{~B}$ shows the results for the symmetric case (both genes $\mathrm{A}$ and $\mathrm{B}$ are self-activating).

Tristability in the Asymmetric TF SATS. We used the above relations between the transcription rates (eq 7) and considered the case that gene $\mathrm{A}$ is regulated by an external input signal $\mathrm{I}$. The 

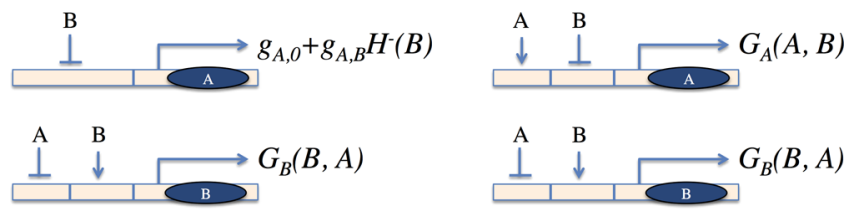

$-A-$

$-B-$

Figure 5. Illustration of the transcription rates for the case of two binding sites. (A) Asymmetric SATS, in which B is self-activating. (B) Symmetric SATS, in which both $A$ and $B$ are self-activating. The $G_{X}$ functions are the formulas for the total transcription rates derived in eq 7.

corresponding deterministic equations for the asymmetric SATS with two binding sites (the noncompetitive case) are:

$$
\begin{aligned}
\mathrm{dA} / \mathrm{d} t & =G_{\mathrm{A}}(\mathrm{I}, \mathrm{B})-k_{\mathrm{A}} \mathrm{A} \\
\mathrm{dB} / \mathrm{d} t & =G_{\mathrm{B}}(\mathrm{B}, \mathrm{A})-k_{\mathrm{B}} \mathrm{B}
\end{aligned}
$$

The corresponding phase space of this model for a specific value of the input signal I when three metastable states coexist is illustrated in Figure 6a, and the bifurcation diagram as a function of the input signal is presented in Figure $6 \mathrm{~b}$. In Figure $6 \mathrm{c}, \mathrm{d}$ we show two examples (for two values of the input signal) of the 1-D effective potential obtained by integrating the equations along the $\mathrm{dA} / \mathrm{d} t=0$ nullcline.

In the SI, we present analyses for the case of one binding site and show that tristability is not observed for this case.
Tristability and Quadristability in the Symmetric TF SATS. For the case of the symmetric TF SATS, the corresponding deterministic equations are:

$$
\begin{aligned}
& \mathrm{dA} / \mathrm{d} t=G_{\mathrm{A}}(\mathrm{A}, \mathrm{B})-k_{\mathrm{A}} \mathrm{A} \\
& \mathrm{dB} / \mathrm{d} t=G_{\mathrm{B}}(\mathrm{B}, \mathrm{A})-k_{\mathrm{B}} \mathrm{B}
\end{aligned}
$$

We found that in this case tristability can be observed for a wider range of circuit parameters than in the asymmetric TF SATS. Interestingly, quadristability can also be observed for some circuit parameters. Note that for this case we did not include an external input signal. The reason is that a consistent inclusion of external signal acting as a TF requires extending the framework presented here for two TFs to the case of three TFs, which is beyond the scope of the current article.

The corresponding phase space of the deterministic eqs 10 for model parameters when three metastable states coexist is illustrated in Figure 7a. In Figure 7b, we show an example of the phase space for model parameters leading to the coexistence of four metastable states - quadristability. The quadristability is a less frequent case because from the dynamic perspective each nullcline should have at least two turns and there should be a total of nine fixed points, in which four are stable, four are saddle, and the other one is unstable.

Chimera Toggle Switch. Building on the generalized model of TF-TF SATS, we derived a generalized model for the miRNATF chimera two-component circuits starting from the miRNA binding/unbinding dynamics discussed in the next section. We

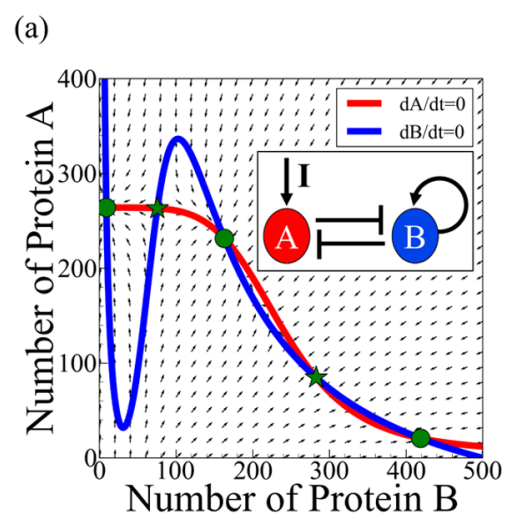

(c)

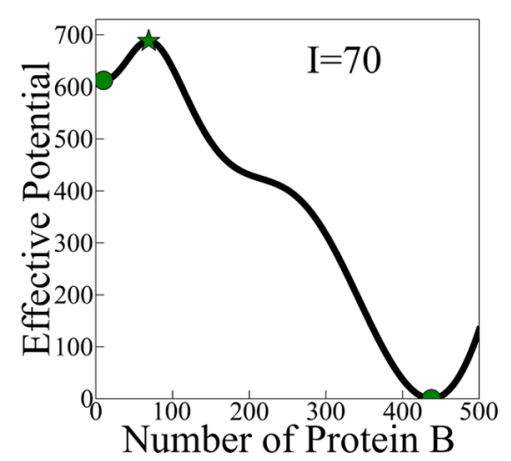

(b)

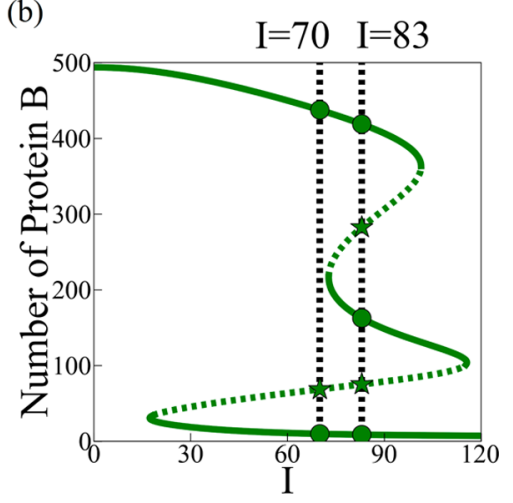

(d)

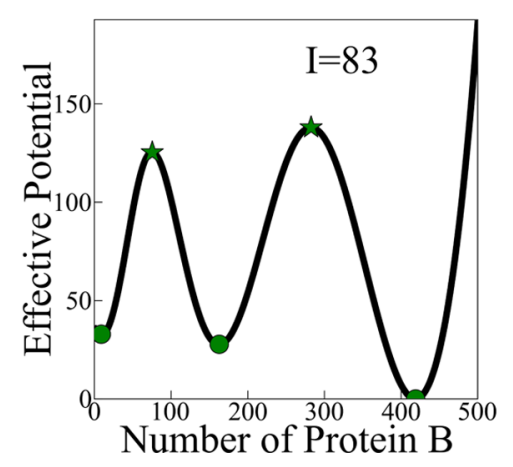

Figure 6. Asymmetric SATS driven by an external signal I. The results shown in this Figure were computed for the schematic circuit depicted in panel A from eq 9. (a) Phase space showing the nullclines for a specific value of the input signal I when three metastable states coexist. (b) Bifurcation diagram as a function of the input signal. The green solid lines represent the stable fixed points, while the green dotted lines represent the unstable fixed points. The two specified cases: two metastable states (the bistability on the left, $I=70$ ) and three coexisting metastable states (tristability on the right, $I=83$ ). Panels $\mathrm{c}$ and $\mathrm{d}$ show a 1-D effective potential computed along the $\mathrm{dA} / \mathrm{dt}=0$ nullcline for $I=70$ and 83 , respectively. Green circles mark the stable fixed points, and the green stars mark unstable saddle fixed points. 
(a)

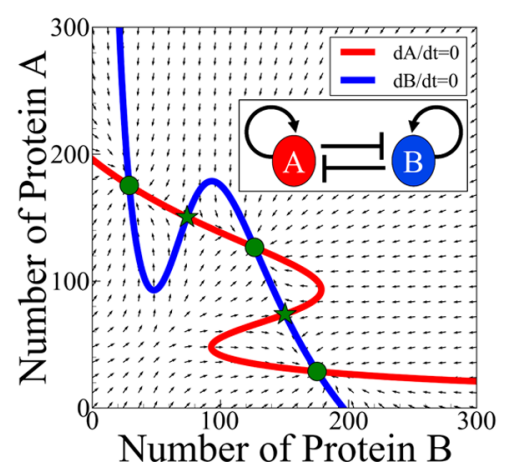

(b)

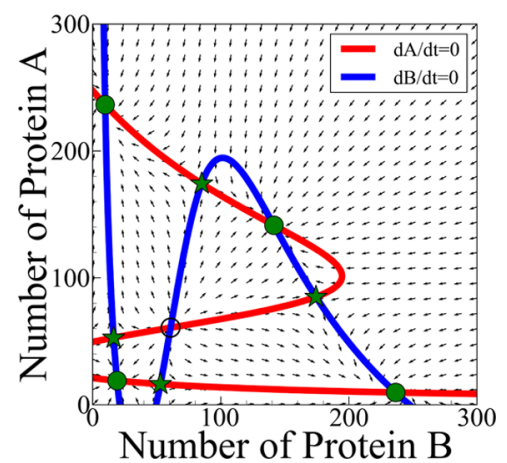

Figure 7. Symmetric SATS phase plane. The results shown in this Figure were computed for the schematic circuit depicted in panel A from eq 10. (a) Phase space showing the nullclines for a specific value of the circuit parameters (SI) when three metastable states coexist (tristability). (b) Phase space showing the nullclines for a specific value of the circuit parameters (SI) when four metastable states coexist (quadristability). Green circles mark stable fixed points, the green stars mark unstable saddle fixed points, and the green diamond marks unstable fixed point.

then present a simple chimera toggle switch driven by an input signal acting as a TF of either the miRNA or the TF. Next, we devise a model for a chimera toggle switch in which the TF is selfactivating and show that three metastable states can exist for some range of the circuit parameters.

Modeling the Chimera Toggle Switch. Schematic presentation of the translation-transcription chimera regulations is depicted in Figure 8A. In Figure 8B we show the miRNA-mRNA binding/unbinding dynamics. We show a specific example of two miRNA binding sites on the mRNA. In general (for $\mathrm{n}$ sites), the translation inhibition depends on the number of the miRNA that are attached and also to which of the sites. When both sites are attached, the inhibition can sometimes be stronger than the product of the inhibition, resulting from binding to each of the sites separately, an example of cooperativity.

We note that although the rate of miRNA unbinding from the mRNA can be low, it has significant effect on the circuit dynamics. To derive the corresponding deterministic equations, we use the following relations:

$$
[\mathrm{m}]+\left[\mu_{1} \mathrm{~m}^{*}\right]+\left[\mu_{2} \mathrm{~m}^{*}\right]+\left[\mu_{12} \mathrm{~m}^{*}\right]=m_{0}
$$

where $[\mathrm{m}],\left[\mu_{1} \mathrm{~m}^{*}\right],\left[\mu_{2} \mathrm{~m}^{*}\right]$, and $\left[\mu_{12} \mathrm{~m}^{*}\right]$ are the concentration of the mRNA at the state $\mathrm{m}$ (free form), $\mu_{1} \mathrm{~m}$ (bounded form in which an miRNA is attached to site one), $\mu_{2} \mathrm{~m}$ (bounded form in which an miRNA is attached to site two), and $\mu_{1} \mu_{2} \mathrm{~m}$ (bounded form in which two miRNAs are attached to both sites one and two), respectively; $m_{0}$ is the total concentration of the mRNA.

Next, to simplify the functional forms of the deterministic equations, we define the following functions (see the SI for the details):

$$
\begin{aligned}
& \mathrm{L}(\mu) \equiv l_{0} \mathrm{M}^{-}(\mu)+\left(l_{1}+l_{2}\right) \mathrm{M}^{+1}(\mu)+l_{12} \mathrm{M}^{+2}(\mu) \\
& \mathrm{Y}_{\mu}(\mu) \equiv\left(\gamma_{\mu 1}+\gamma_{\mu 2}\right) \mathrm{M}^{+1}(\mu)+2 \gamma_{\mu 12} \mathrm{M}^{+2}(\mu) \\
& \mathrm{Y}_{\mathrm{m}}(\mu) \equiv\left(\gamma_{\mathrm{m} 1}+\gamma_{\mathrm{m} 2}\right) \mathrm{M}^{+1}(\mu)+\gamma_{\mathrm{m} 12} \mathrm{M}^{+2}(\mu)
\end{aligned}
$$

where the $M$ functions are defined as

$$
\begin{aligned}
& \mathrm{M}^{-}(\mu) \equiv 1 /\left[\left(1+\left(\mu / \mu_{0}\right)\right]^{2}\right. \\
& \mathrm{M}^{+1}(\mu) \equiv\left(\mu / \mu_{0}\right) /\left[\left(1+\left(\mu / \mu_{0}\right)\right]^{2}\right. \\
& \mathrm{M}^{+2}(\mu) \equiv\left(\mu / \mu_{0}\right)^{2} /\left[\left(1+\left(\mu / \mu_{0}\right)\right]^{2}\right.
\end{aligned}
$$

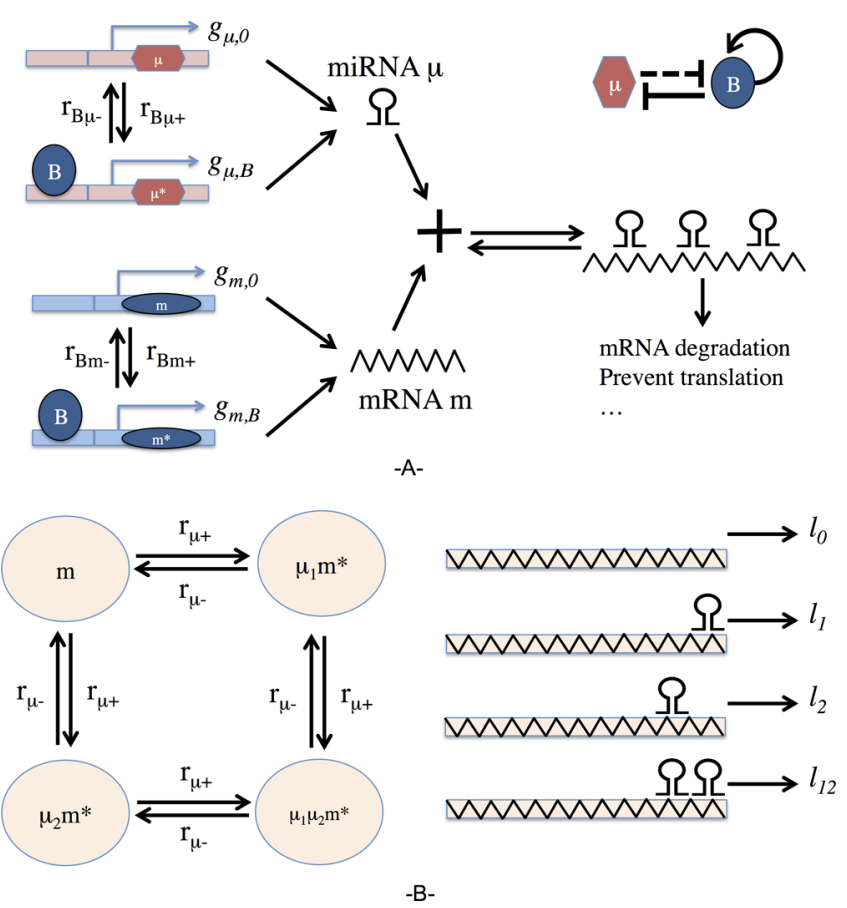

Figure 8. Illustration of the chimera SATS binding-unbinding dynamics. (A) Illustration of the dynamics of the circuits on the molecular level. The miRNA-TF chimera switch is presented in the upper right part. The miRNA $\mu$ 's (in red hexagon) transcription is repressed by the TF B (in blue ellipse). Note that, in general, gene B is also either self-activating or self-inhibiting. Meanwhile, one or several miRNA $\mu$ bind to the mRNA $\mathrm{m}$ to silence the translation of the protein $\mathrm{B}$ because the miRNA can either facilitate mRNA degradation or inhibit translation. (B) Illustration of the binding-unbinding dynamics between an mRNA and two miRNAs. The left panel shows chemical reactions of the binding/unbinding events among the four mRNA states: the free form $\mathrm{m}$, the miRNA-bounded forms $\mu_{1} \mathrm{~m} \mu_{2} \mathrm{~m}$ when an miRNA binds either the first or the second site, and the miRNAbounded form $\mu_{1} \mu_{2} \mathrm{~m}$ when two miRNAs bind to both sites. The binding rate $r_{\mu^{+}}$and the unbinding rates $r_{\mu-}$ are the same for all reactions. The right part illustrates the translation rates for each promoter state, denoted by $l_{0}, l_{1}, l_{2}$, and $l_{12}$.

In the SI, we present the general results for the case that the mRNA has $n$ binding sites $(n>2)$ for the miRNAs.

Analysis of a Simple Chimera Toggle Switch Driven by an External Signal. Using the above definitions, the deterministic 

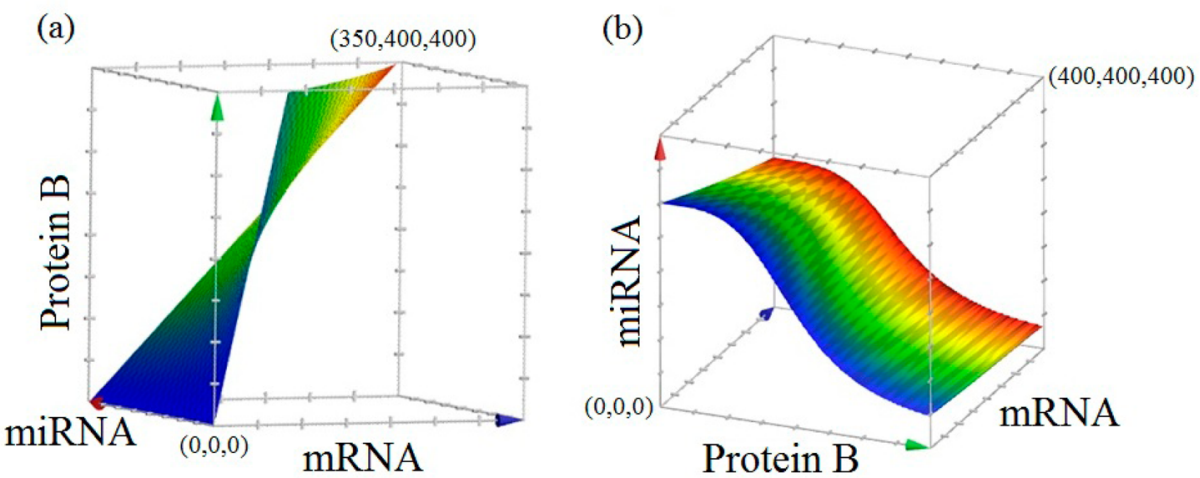

Figure 9. Illustration of the null-surface of the 3-D phase space describing the simple chimera toggle switch. (a) Null surface defined by $\mathrm{d} B / \mathrm{d} t=0$. (b) Null surface defined by $\mathrm{d} \mu / \mathrm{d} t=0$. In each panel, the colors on the surface represent the molecule level (it increases from blue to red) for the mRNA axis.

(a)

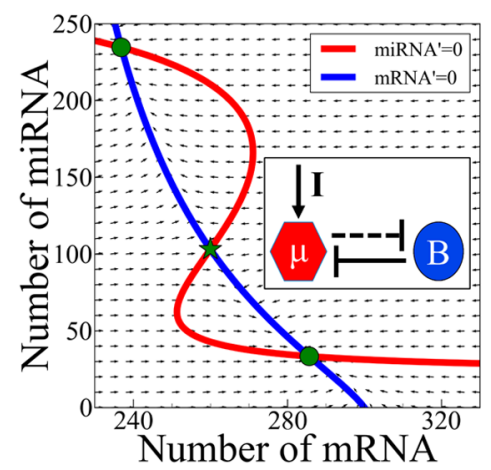

(c)

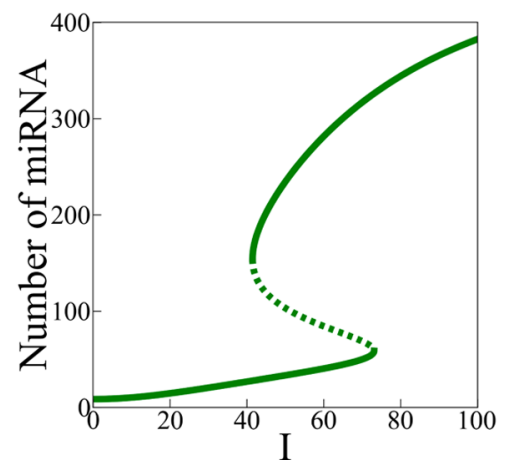

(b)

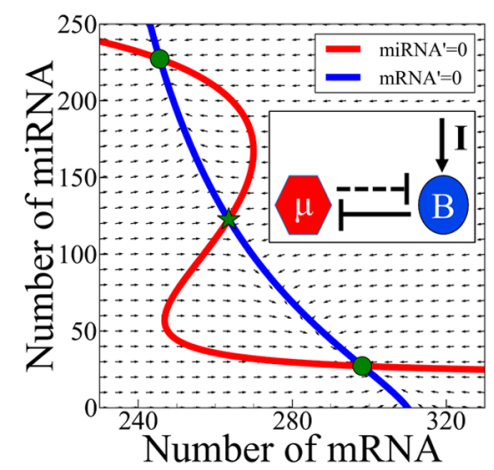

(d)

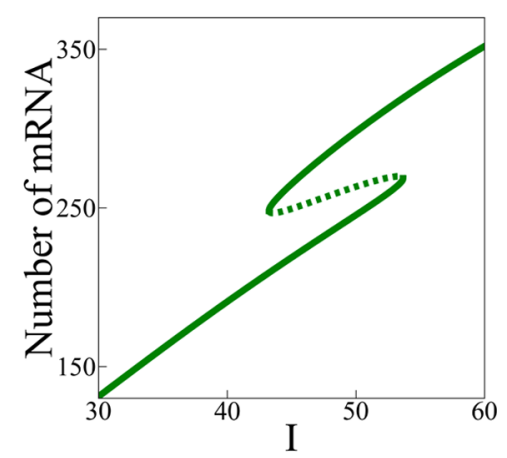

Figure 10. Simple chimera toggle switch driven by an external signal I. The results shown in this Figure were computed for the schematic circuits depicted in panel a, in which the miRNA is driven by the input signal, and panel $b$, in which gene B is driven by the input signal, using eqs 14 and 15 , respectively. In both Figures, we show 2-D mRNA-miRNA phase space for $\mathrm{dB} / \mathrm{d} t=0$. We note that the nullclines and the arrows (showing the flow in the phase space) are projected from the null-surface $\mathrm{dB} / \mathrm{d} t=0$ shown in Figure $9 \mathrm{~b}$ on the mRNA-miRNA (m- $\mu$ Euclidean 2-D space for $\mathrm{B}=0)$. The corresponding bifurcation diagrams as a function of the driving input signal I are shown in panels $\mathrm{c}$ and d, respectively.

equations for a simple chimera switch when the miRNA is transcriptionally driven by an input signal I are:

$$
\begin{aligned}
& \mathrm{d} \mu / \mathrm{d} t=G_{\mu}(\mathrm{I}, \mathrm{B})-\mathrm{mY}_{\mu}(\mu)-k_{\mu} \mu \\
& \mathrm{dm} / \mathrm{d} t=g_{\mathrm{m}, 0}-\mathrm{mY}_{\mathrm{m}}(\mu)-k_{\mathrm{m}} \mathrm{m} \\
& \mathrm{dB} / \mathrm{d} t=\mathrm{mL}(\mu)-k_{\mathrm{B}} \mathrm{B}
\end{aligned}
$$

The deterministic equations for a simple chimera switch when gene $\mathrm{B}$ is transcriptionally driven by an input signal I are:

$$
\begin{aligned}
& \mathrm{d} \mu / \mathrm{d} t=\mathrm{g}_{\mu, 0}+g_{\mu, \mathrm{B}} \mathrm{H}^{-}(\mathrm{B})-\mathrm{mY}_{\mu}(\mu)-k_{\mu} \mu \\
& \mathrm{dm} / \mathrm{d} t=g_{\mathrm{m}, 0}+g_{\mathrm{m}, \mathrm{I}} \mathrm{H}^{+}(\mathrm{I})-\mathrm{mY}_{\mathrm{m}}(\mu)-k_{\mathrm{m}} \mathrm{m} \\
& \mathrm{dB} / \mathrm{d} t=\mathrm{mL}(\mu)-k_{\mathrm{B}} \mathrm{B}
\end{aligned}
$$

It is important to emphasize that this approach incorporates key features that were not presented in previous models. ${ }^{26,27,34-36}$ First, the model is able to include the effects of translation silencing by multiple binding sites of miRNA on the mRNA. As shown in the SI, the effect of multiple binding of miRNA differs from the cooperative effect in the case of TFs. Therefore, this effect cannot be modeled in terms of the standard Hill functions 
that are traditionally used to model transcriptional regulation. Our derivation shows that the Hill functions are replaced by the new $M$ functions that capture the nonlinearity of the process. Second, the model contains explicitly a new translational inhibition term, $\mathrm{L}(\mu)$. Third, all of the terms for the translation inhibition and the miRNA-assisted degradation are derived from the binding/unbinding chemical reactions between miRNA and mRNA. Fourth, the resulting dynamics is described by three coupled deterministic equations, unlike the previously presented model. $^{26}$

The three-coupled deterministic equations of the new model for miRNA-TF chimera circuits define a 3-D phase space. This contrasts with the case of TF-TF circuits whose deterministic dynamics is described by two coupled equations defining a 2-D phase space. When it comes to $3-\mathrm{D}$ phase space, the nullclines are replaced by null-surfaces. In the case of the simple chimera toggle switch, while each of the equations $\mathrm{d} \mu / \mathrm{d} t=0$ and $\mathrm{dB} / \mathrm{d} t=0$ define a null-surface, the equation $\mathrm{dm} / \mathrm{d} t=0$ is degenerated and defines a nullcline because the equation depends only on the mRNAs and the miRNAs. This degeneracy is changed when $B$ is self-activating because the equation $\mathrm{dm} / \mathrm{d} t=0$ also depends on the level of $\mathrm{B}$. Therefore, $\mathrm{dm} / \mathrm{d} t=0$ defines a null-surface as is discussed further below.

In Figure 9, we show the null-surface for $\mathrm{d} \mu / \mathrm{d} t=0$ and $\mathrm{dB} / \mathrm{dt}$ $=0$ for eqs 14 and 15 in the limit $\mathrm{I}=0$ (so the two set of equations are the same when $\mathrm{I}=0$ ). The $2-\mathrm{D}$ phase space for $\mathrm{dB} / \mathrm{d} t=0$, projected on the $\mathrm{m}-\mu$ Euclidean 2-D space of the chimera toggle switch is shown in Figure 10a for the miRNA driven by the external signal case and in Figure 10c for the gene B driven by the external signal case. Both Figures are shown for specific values of the input signal when two metastable states coexist. The corresponding bifurcation diagrams as a function of the driving input signal I are shown in Figure 10c,d respectively.

Self-Activating Chimera Toggle Switch Driven by an External Signal. The deterministic equations for this case, when the external signal acts as a TF on the miRNA, are:

$$
\begin{aligned}
\mathrm{d} \mu / \mathrm{d} t & =G_{\mu}(\mathrm{I}, \mathrm{B})-\mathrm{mY}_{\mu}(\mu)-k_{\mu} \mu \\
\mathrm{dm} / \mathrm{d} t & =g_{\mathrm{m}, 0}+g_{\mathrm{m}, \mathrm{B}} \mathrm{H}^{+}(\mathrm{B})-\mathrm{mY}_{\mathrm{m}}(\mu)-k_{\mathrm{m}} \mathrm{m} \\
\mathrm{dB} / \mathrm{d} t & =\mathrm{mL}(\mu)-k_{\mathrm{B}} \mathrm{B}
\end{aligned}
$$

The corresponding phase space of this model for a specific value of the input signal when three metastable states coexist is illustrated in Figure 11a. The corresponding bifurcation diagrams of the miRNAs, of the mRNAs and of the protein $\mathrm{B}$ as a function of the driving input signal I are shown in Figure 10b-d.

\section{DISCUSSION}

SATSs serve as core two-component circuits in many decision modules, especially related to cell differentiation during embryonic development and tumorigenesis. They are the simplest possible circuits with the ability of making a decision. Typically these components can comprise either two mutually inhibiting genes (TFs) with one or both of the genes also being self-activating or an miRNA-TF chimera pair in which the gene is self-activating. A well-studied example of TF-TF SATS is the (CDX2-OCT3/4) pair, ${ }^{5}$ which is associated with the differentiation of ES cells. The miRNA-TF chimera SATS play an important role in the context of cancer. Well-studied examples are the (let-7-Lin28) pair associated with regulation of selfrenewal of cancer stem cells and the (miR200-ZEB1/2) pair associated with EMT and the reverse transitions (MET). ${ }^{15,16,20}$ (a)

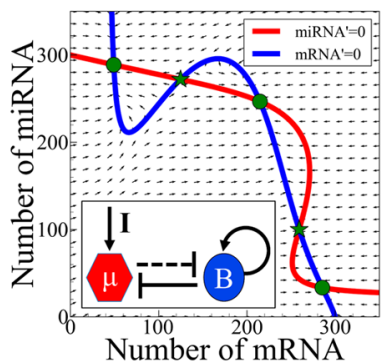

(c)

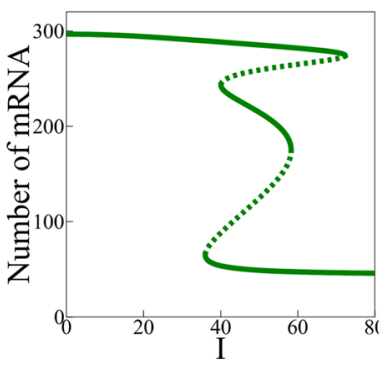

(b)

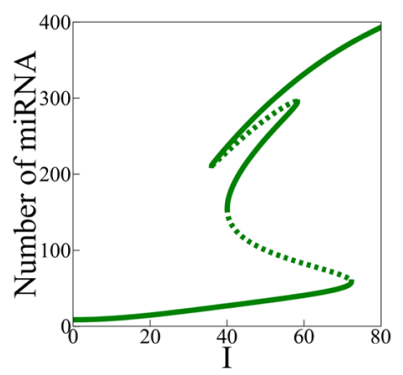

(d)

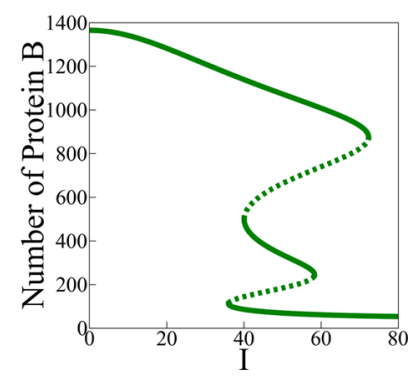

Figure 11. Asymmetric self-activating chimera toggle switch driven by an external signal I. The results shown in this Figure were computed for the schematic circuits depicted in Figure 1a, in which the miRNA is driven by the input signal and gene $B$ is self-activating, using eq 16. (a) 2 $\mathrm{D}$ mRNA-miRNA phase space for $\mathrm{dB} / \mathrm{d} t=0$. We note that the nullclines and the arrows (showing the flow in the phase space) are projected from the nullsurface $\mathrm{dB} / \mathrm{d} t=0$ on the mRNA-miRNA (m- $\mu$ Euclidian 2-D space for $B=0$. The corresponding bifurcation diagrams of the miRNAs, of the mRNAs, and of the protein $B$ as a function of the driving input signal I are shown in Figure $10 \mathrm{~b}-\mathrm{d}$, respectively.

Previous studies of TF-TF SATS used a variety of deterministic equations. ${ }^{6-8,37}$ We devised a theoretical framework of the SATSs by starting from the detailed TF-promoter binding/unbinding dynamics for both the noncompetitive case (two binding sites, one for each TF) and the competitive case (only one binding site for both TFs). We also extended the previous studies by including an external input signal $\mathrm{I}$ that mimics driving the SATS by another circuit. Therefore, the action of the driving input signal was included as an additional TF I. For complete modeling, we have also studied the miRNA-TF chimera SATSs starting from the miRNA-mRNA binding/ unbinding dynamics. The three-coupled deterministic equations obtained by our approach qualitatively differ from the deterministic equations previously used to study chimera toggle switch without self-activation. ${ }^{26}$

We studied four cases of TF-TF SATSs - the asymmetric and symmetric self-activation, for the cases of noncompetitive binding and competitive binding. (The promoter has two binding sites and one binding site, respectively.) We found that SATS can exhibit tristability - the coexistence of three metastable states - for a biologically reasonable range of the circuit parameters (SI). The only exception is asymmetric SATS with competitive binding, which can only have bistability.

For tristability, two of the metastable states correspond to the two states of the simple toggle switch described in the Introduction. These are the $(0,1)$ and the $(1,0)$ states in which gene $A$ has low expression and gene $B$ is highly expressed and vice versa. The third metastable state is an intermediate state corresponding to state $(1 / 2,1 / 2)$ in which both genes $\mathrm{A}$ and $\mathrm{B}$ have intermediate levels of expression. We also included 
bifurcation analysis as a function of the input signal to show that the intermediate state has a finite but significant range of existence. We found that the symmetric SATS can have quadristability (coexistence of four metastable states), with three of the metastable states similar to the ones in the tristability, and the additional one corresponds to $(0,0)$ state.

The main motivation of the article is to show that the miRNATF self-activating chimera toggle switches can also give rise to tristability. We focused on the asymmetric case in which the gene is self-activating and studied the competitive and noncompetitive cases with the input signal driving either the miRNA or the gene. Dynamical system analyses revealed that the chimera SATSs also exhibit tristability. Moreover, the dynamics of the miRNA-TF toggle switch is qualitatively different from that of the TF-TF toggle switch because the nonlinearity in translational repression functions $\mathrm{L}(\mu), \mathrm{Y}_{\mathrm{m}}(\mu)$, and $\mathrm{Y}_{\mu}(\mu)$ is different from that in Hills' repression functions $\mathrm{H}^{-}(\mathrm{A})$.

These results offer novel insights into recent experiments in cell fate determination in cancer. For instance, a "metastable" intermediate state of the cell has been seen during EMT. This state reverts back to an epithelial phenotype (one of the steady states) upon the removal of TGF- $\beta .^{24}$ It is accompanied by reduced proliferation and decreased transcription of the cell cycle proteins, thus indicating dormancy. ${ }^{38,39}$ Also, the intermediate state shows many features of mesenchymal phenotype (the other steady state), such as loss of various cell-cell junctions, cell polarity, and weakened attachment to basement membrane. ${ }^{38}$ Besides, it is associated with a higher production of inflammatory cytokines IL-6 and IL-11 $1^{38,40}$ and a different repertoire of autocrine survival networks than in the epithelial state. ${ }^{38,41,42}$ Thus, this intermediate state of EMT is a transient phenotype observed when cells exit the epithelial state but have not completely attained the mesenchymal state.

The activation of EMT has been associated with acquiring what are frequently termed in the literature as stemness traits or "cancer stem cell" (CSCs) traits by normal mammary epithelial cells and neoplastic cells. ${ }^{43-45}$ Furthermore, the reverse of EMT, MET, is the first step that occurs during reprogramming of differentiated cells to iPSCs. ${ }^{46,47}$ Also, another group showed that the mesenchymal state has reduced self-renewing properties. $^{48}$ Put together, it is suggestive that the regulations of EMTMET and stemness are linked together through molecular links that are not yet completely deciphered and understood.

The notion of elevated stemness (programmability) in special cancer cells - the existence of CSCs - is a subject of vigorous debate. ${ }^{49}$ What is becoming commonly agreed upon is that the subpopulations of special tumorigenic (tumor-forming) cancer cells exist within both tumors and hematological cancers that possess the ability of renewal and differentiation into all (or most as well as new) cancer cell types in the particular cancer. ${ }^{50,51}$ It is also widely agreed that these subpopulations can successfully evade, aided by their dormancy, cancer treatments (chemo, radiation, and targeted) and cause relapse and metastasis by giving rise to new tumors. ${ }^{52,53}$ The reason for using the term CSC originated from the fact that these subpopulations share several features with normal stem cells and induced pluripotent stem cells. ${ }^{54}$ We prefer to use the term PDCCs because dormancy is an essential aspect of these cells.

The existence of intermediate states in the chimera SATSs might be associated with a putative mechanism of initial transition into a pre-PDCC phenotype - cells with the potential to become PDCC. The idea is that under special circumstances (special value of the input signals) the decision circuit (e.g., the
miR200-Zeb1/2 chimera pair) is kept in the intermediate state for a sufficiently long time and therefore is able to trigger a cascade of transition of downstream decision modules. Theoretical investigations of this possibility will require studying the dynamics of decision modules comprising interacting twocomponent and three-component circuits as well as the interactions between different modules.

Another important extension of this work is to study the interplay between cell fate circuits and cell-cell interaction circuits such as the Notch-Delta system, which plays a crucial role in cell-fate determination during embryonic development and tumorigenesis. ${ }^{55,56}$ A plausible hypothesis is that cells become PDCC only in the rare cooperative case when several of their neighboring cells enter into the intermediate state - the prePDCC state - at the same time. The idea is that the intermediate state triggers the Notch-Delta to form an interaction between neighboring cells in the pre-PDCC state. This, in turn, acts as a positive feedback on the cell fate circuits to complete the transition into the PDCC state. Recent experimental studies ${ }^{57}$ revealed an interesting link between the miR200-ZEB1/2 chimera switch and the Notch-Delta system, supporting the above hypothesis. This and other examples call for extending the dynamical system investigations presented here by coupling the intracellular dynamics of each cell to cell-cell interactions.

\section{ASSOCIATED CONTENT}

\section{Supporting Information}

Generalized model of transcriptional regulation by two TFs, the generalized model for the chimera toggle switch, modeling results for the SATS with the one-binding-site model, and list of the model parameters. This information is available free of charge via the Internet at http://pubs.acs.org.

\section{AUTHOR INFORMATION}

\section{Corresponding Author}

*E-mail: eshel@rice.edu; Phone: 713-348-8162; Fax: 713-3488120 (E.B.-J.). E-mail: mingyang.lu@rice.edu; Phone: 713-3488168 (M.L.).

\section{Notes}

The authors declare no competing financial interest.

\section{ACKNOWLEDGMENTS}

We thank Dr. Peter Wolynes for collaboration on bacteria decision making and many important insights. We thank Dr. Herbert Levine for valuable discussions regarding the modeling aspects. E.B.-J. is most thankful to Dr. Mary F. McGuire, Dr. Robert E. Brown, and Dr. Donald S. Coffey for many enlightening conversations regarding the biochemistry, cancer biology and therapeutic aspects associated with various regulatory pathways. This research has been supported by the Center for Theoretical Biological Physics sponsored by the NSF (Grant PHY-1308264), by the Cancer Prevention and Research Institute of Texas (CPRIT) at Rice University, and the Tauber Family Foundation and the Maguy-Glass Chair in Physics of Complex Systems at Tel Aviv University and by the NSF INSPIRE award MCB-1241332.

\section{REFERENCES}

(1) Laslo, P.; Spooner, C. J.; Warmflash, A.; Lancki, D. W.; Lee, H. J.; Sciammas, R.; Gantner, B. N.; Dinner, A. R.; Singh, H. Multilineage Transcriptional Priming and Determination of Alternate Hematopoietic Cell Fates. Cell 2006, 126, 755-766. 
(2) Kim, M. S.; Kim, J. R.; Kim, D.; Lander, A. D.; Cho, K. H. Spatiotemporal Network Motif Reveals the Biological Traits of Developmental Gene Regulatory Networks in Drosophila Melanogaster. BMC Syst. Biol. 2012, 6, 31.

(3) Schultz, D.; Wolynes, P. G.; Ben Jacob, E.; Onuchic, J. N. Deciding Fate in Adverse Times: Sporulation and Competence in Bacillus Subtilis. Proc. Natl. Acad. Sci. U.S.A. 2009, 106, 21027-21034.

(4) Duff, C.; Smith-Miles, K.; Lopes, L.; Tian, T. Mathematical Modelling of Stem Cell Differentiation: The PU.1-GATA-1 Interaction. J. Math. Biol. 2012, 64, 449-468.

(5) Niwa, H.; Toyooka, Y.; Shimosato, D.; Strumpf, D.; Takahashi, K.; Yagi, R; Rossant, J. Interaction between Oct3/4 and Cdx2 Determines Trophectoderm Differentiation. Cell 2005, 123, 917-929.

(6) Guantes, R.; Poyatos, J. F. Multistable Decision Switches for Flexible Control of Epigenetic Differentiation. PLoS Comput. Biol. 2008, 4, e1000235.

(7) Macia, J.; Widder, S.; Sole, R. Why are Cellular Switches Boolean? General Conditions for Multistable Genetic Circuits. J. Theor. Biol. 2009, 261, 126-135.

(8) Wang, J.; Zhang, K.; Xu, L.; Wang, E. Quantifying the Waddington Landscape and Biological Paths for Development and Differentiation. Proc. Natl. Acad. Sci. U.S.A. 2011, 108, 8257-8262.

(9) Bintu, L.; Buchler, N. E.; Garcia, H. G.; Gerland, U.; Hwa, T.; Kondev, J.; Kuhlman, T.; Philips, R. Transcriptional Regulation by the Numbers: Applications. Curr. Opin. Genet. Dev. 2005, 15, 125-135.

(10) Walczak, A. M.; Sasai, M.; Wolynes, P. G. Self-Consistent Proteomic Field Theory of Stochastic Gene Switches. Biophys. J. 2005, $88,828-850$.

(11) Sasai, M.; Wolynes, P. G. Stochastic Gene Expression as a ManyBody Problem. Proc. Natl. Acad. Sci. U.S.A. 2003, 100, 2374-2379.

(12) Ivey, K. N.; Srivastava, D. MicroRNAs as Regulators of Differentiation and Cell Fate Decisions. Cell Stem Cell 2010, 7, 36-41.

(13) Esquela-Kerscher, A.; Slack, F. J. Oncomirs - MicroRNAs with a Role in Cancer. Nat. Rev. Cancer 2006, 6, 259-269.

(14) Martinez, N. J.; Walhout, A. J. The Interplay between Transcription Factors and MicroRNAs in Genome-Scale Regulatory Networks. Bioessays 2009, 31, 435-45.

(15) Yang, X.; Lin, X.; Zhong, X.; Kaur, S.; Liang, S.; Lassus, H.; Wang, L.; Katsaros, D.; Montone, K.; Zhao, X.; et al. Double-Negative Feedback Loop between Reprogramming Factor LIN28 and microRNA let-7 Regulates Aldehyde Dehydrogenase 1-Positive Cancer Stem Cells. Cancer Res. 2010, 70, 9463-9472.

(16) Bracken, C. P.; Gregory, P. A.; Kolesnikoff, N.; Bert, A. G.; Wang, J.; Shannon, M. F.; Goodall, G. J. a Double-Negative Feedback Loop Between ZEB1-SIP1 and the microRNA-200 Family Regulates Epithelial-Mesenchymal Transition. Cancer Res. 2008, 68, 7846-7854.

(17) Siemens, H.; Jackstadt, R.; Hunten, S.; Kaller, M.; Menssen, A.; Gotz, U.; Hermeking, H. miR-34 and SNAIL Form a Double-Negative Feedback Loop to Regulate Epithelial-Mesenchymal Transitions. Cell Cycle 2011, 10, 4256-4271.

(18) Yang, Y.; Ahn, Y. H.; Gibbons, D. L.; Zang, Y.; Lin, W.; Thilaganathan, N.; Alvarez, C. A.; Moreira, D. C.; Creighton, C. J.; Gregory, P. A.; et al. The Notch ligand Jagged2 promotes lung adenocarcinoma metastasis through a miR-200-dependent pathway in mice. J. Clin. Invest. 2011, 121, 1373-1385.

(19) Qian, P.; Banerjee, A.; Wu, Z. S.; Zhang, X.; Wang, H.; Pandey, V.; Zhang, W. J.; Lv, X. F.; Tan, S.; Lobie, P. E.; et al. Loss of SNAIL regulated miR-128-2 on chromosome 3p22.3 Targets Multiple Stem Cell Factors to Promote Transformation of Mammary Epithelial Cells. Cancer Res. 2012, 72, 6036-6050.

(20) Burk, U.; Schubert, J.; Wellner, U.; Schmalhofer, O.; Vincan, E.; Spaderna, S.; Brabletz, T. A reciprocal repression between ZEB1 and members of the miR-200 family promotes EMT and Invasion in Cancer Cells. EMBO Rep. 2008, 9, 582-589.

(21) Hill, L.; Browne, G.; Tulchinsky, E. ZEB/miR-200 Feedback Loop: At the Crossroads of Signal Transduction in Cancer. Int. J. Cancer 2013, 132, 745-754.
(22) Polytarchou, C.; Iliopoulos, D.; Struhl, K. An Integrated Transcriptional Regulatory Circuit That Reinforces the Breast Cancer Stem Cell State. Proc. Natl. Acad. Sci. U.S.A. 2012, 109, 14470-14475.

(23) Chickarmane, V.; Troein, C.; Nuber, U. A.; Sauro, H. M.; Peterson, C. Transcriptional Dynamics of the Embryonic Stem Cell Switch. PLoS Comput. Biol. 2006, 2, 1080-1092.

(24) Gregory, P. A.; Bracken, C. P.; Smith, E.; Bert, A. G.; Wright, J. A.; Roslan, S.; Morris, M.; Wyatt, L.; Farshid, G.; Lim, Y. Y.; et al. An Autocrine TGF-beta/ZEB/miR-200 Signaling Network Regulates Establishment and Maintenance of Epithelial-Mesenchymal Transition. Mol. Biol. Cell 2011, 22, 1686-1698.

(25) Choi, Y. J.; Lin, C. P.; Ho, J. J.; He, X.; Okada, N.; Bu, P.; Zhong, Y.; Kim, S. Y.; Bennett, M. J.; Chen, C.; et al. miR-34 miRNAs Provide a Barrier for Somatic Cell Reprogramming. Nat. Cell Biol. 2011, 13, $1353-1360$

(26) Zhou, P.; Cai, S.; Li, Z.; Wang, R. Mechanisms Generating Bistability and Oscillations in microRNA-Mediated Motifs. Phys. Rev. E: Stat., Nonlinear, Soft Matter Phys. 2012, 85, 041916.

(27) Levine, E.; Ben Jacob, E.; Levine, H. Target-Specific and Global Effectors in Gene Regulation by MicroRNA. Biophys. J. 2007, 93, L52L54

(28) Liew, C. W.; Rand, K. D.; Simpson, R. J.; Yung, W. W.; Mansfield, R. E.; Crossley, M.; Proetorius-Ibba, M.; Nerlov, C.; Poulsen, F. M.; Mackay, J. P. Molecular Analysis of the Interaction between the Hematopoietic Master Transcription Factors GATA-1 and PU.1. J. Biol. Chem. 2006, 281, 28296-28306.

(29) Ceppi, P.; Peter, M. E. MicroRNAs Regulate Both Epithelial-toMesenchymal Transition and Cancer Stem Cells. Oncogene 2013, 1-10.

(30) Lamouille, S.; Subramanyam, D.; Blelloch, R.; Derncyk, R. Regulation of Epithelial-Mesenchymal and Mesenchymal-Epithelial Transitions by microRNAs. Curr. Opin. Cell Biol. 2013, 25, 200-207.

(31) Sanchez-Tillo, E.; Liu, Y.; de Barrios, O.; Siles, L.; Fanlo, L.; Cuatrecasas, M.; Darling, D. S.; Dean, D. C.; Castells, A.; Postigo, A. EMT-Activating Transcription Factors in Cancer: Beyond EMT and Tumor Invasiveness. Cell. Mol. Life Sci. 2012, 69, 3429-3456.

(32) Peinado, H.; Oldema, D.; Cano, A. Snail, Zeb and bHLH Factors in Tumour Progression: An Alliance against the Epithelial Phenotype? Nat. Rev. Cancer 2007, 7, 415-428.

(33) Shimono, Y.; Zabala, M.; Cho, R. W.; Lobo, N.; Dalerba, P.; Qian, D.; Diehn, M.; Liu, H.; Panula, S. P.; Chiao, E.; et al. Downregulation of miRNA-200c Links Breast Cancer Stem Cells with Normal Stem Cells. Cell 2009, 138, 592-603.

(34) Zhdanov, V. P. Bistability in Gene Transcription: Interplay of Messenger RNA, Protein, And Nonprotein Coding RNA. Biosystems 2009, 95, 75-81.

(35) Shimoni, Y.; Friedlander, G.; Hetzroni, G.; Niv, G.; Altuvia, S.; Biham, O.; Margalit, H. Regulation of Gene Expression by Small NonCoding RNAs: a Quantitative View. Mol. Syst. Biol. 2007, 3, 138.

(36) Liu, D.; Chang, X.; Liu, Z.; Chen, L.; Wang, R. Bistability and Oscillations in Gene Regulation Mediated by Small Noncoding RNAs. PLoS One 2011, 6, e17029.

(37) Andrecut, M.; Halley, J. D.; Winkler, D. A.; Huang, S. a General Model for Binary Cell Fate Decision Gene Circuits with Degeneracy: Indeterminacy and Switch Behavior in the Absence of Cooperativity. PLoS One 2011, 6, e19358.

(38) Thomson, S.; Petti, F.; Sujka-Kwok, I.; Mercado, P.; Bean, J.; Monaghan, M.; Seymour, S. L.; Argast, G. M.; Epstein, D. M.; Haley, J. D. A Systems View of Epithelial-Mesenchymal Transition Signaling States. Clin. Exp. Metastasis. 2011, 28, 137-155.

(39) Argast, G. M.; Mercado, P.; Mulford, I. J.; O’Connor, M.; Keane, D. M.; Shaaban, S.; Epstein, D. M.; Pachter, J. A.; Kan, J. L. Cooperative Signaling between Oncostatin M, Hepatocyte Growth Factor and Transforming Growth Factor-Beta Enhances Epithelial to Mesenchymal Transition in Lung and Pancreatic Tumor Models. Cells Tissues Organs 2011, 193, 114-132.

(40) Thomson, S.; Petti, F.; Sujka-Kwok, I.; Epstein, D.; Haley, J. D. Kinase Switching in Mesenchymal-Like Non-Small Cell Lung Cancer Lines Contributes to EGFR Inhibitor Resistance through Pathway Redundancy. Clin. Exp. Metastasis 2008, 25, 843-854. 
(41) Gjerdrum, C.; Tiron, C.; Høiby, T.; Stefansson, I.; Haugen, H.; Sandal, T.; Collett, K.; Li, S.; McCormack, E.; Gjertsen, B. T.; et al. Axl is an Essential Epithelial-to-Mesenchymal Transition-Induced Regulator of Breast Cancer Metastasis and Patient Survival. Proc. Natl. Acad. Sci. U.S.A. 2010, 107, 1124-1129.

(42) Gao, S. P.; Mark, K. G.; Leslie, K.; Pao, W.; Motoi, N.; Gerald, W. L.; Travis, W. D.; Bornmann, W.; Veach, D.; Clarkson, B.; et al. Mutations in the EGFR Kinase Domain Mediate STAT3 Activation via IL-6 Production in Human Lung Adenocarcinomas. J. Clin. Invest. 2007, $117,3846-3856$.

(43) Mani, S. A.; Guo, W.; Liao, M. J.; Eaton, E. N.; Ayyanan, A.; Zhou, A. Y.; Brooks, M.; Reinhard, F.; Zhang, C. C.; Shipitsin, M.; et al. The Epithelial-Mesenchymal Transition Generates Cells with Properties of Stem Cells. Cell 2008, 133, 704-715.

(44) Morel, A. P.; Lièvre, M.; Thomas, C.; Hinkal, G.; Ansieau, S.; Puisieux, A. Generation of Breast Cancer Stem Cells through EpithelialMesenchymal Transition. PLoS One 2008, 3, e2888.

(45) Scheel, C.; Weinberg, R. A. Cancer Stem Cells and EpithelialMesenchymal Transition: Concepts and Molecular Links. Semin. Cancer Biol. 2012, 22, 396-403.

(46) Li, R.; Liang, J.; Ni, S.; Zhou, T.; Qing, X.; Li, H.; He, W.; Chen, J.; Li, F.; Zhuang, Q.; et al. A Mesenchymal-to-Epithelial Transition Initiates and Is Required for the Nuclear Reprogramming of Mouse Fibroblasts. Cell Stem Cell 2010, 7, 51-63.

(47) Samavarchi-Tehrani, P.; Golipour, A.; David, L.; Sung, H. K.; Beyer, T. A.; Datti, A.; Woltjen, K.; Nagy, A.; Wrana, J. L. Functional Genomics Reveals a BMP-Driven Mesenchymal-to-Epithelial Transition in the Initiation of Somatic Cell Reprogramming. Cell Stem Cell 2010, 7, 64-77.

(48) Celià-Terrassa, T.; Meca-Cortés, O.; Mateo, F.; de Paz, A. M.; Rubio, N.; Arnal-Estapé, A.; Ell, B. J.; Bermudo, R.; Díaz, A.; GuerraRebollo, M.; et al. Epithelial-Mesenchymal Transition Can Suppress Major Attributes of Human Epithelial Tumor-Initiating Cells. J. Clin. Invest. 2012, 122, 1849-1868.

(49) Gupta, P. B.; Chaffer, C. L.; Weinberg, R. A. Cancer Stem Cells: Mirage or Reality? Nat. Med. 2009, 15, 1010-1012.

(50) Azizi, E.; Wicha, M. S. Point: Cancer Stem Cells-The Evidence Accumulates. Clin. Chem. 2013, 59, 205-207.

(51) Kirk, R. Tumour Evolution: Evidence Points to the Existence of Cancer Stem Cells. Nat. Rev. Clin. Oncol. 2012, 9, 552.

(52) Izumiya, M.; Kabashima, A.; Higuchi, H.; Igarashi, T.; Sakai, G.; Iizuka, H.; Nakamura, S.; Adachi, M.; Hamamoto, Y.; Funakoshi, S.; et al. Chemoresistance Is Associated with Cancer Stem Cell-Like Properties and Epithelial-to-Mesenchymal Transition in Pancreatic Cancer Cells. Anticancer Res. 2012, 32, 3847-3853.

(53) Chuthapisith, S.; Eremin, J.; El-Sheemey, M.; Eremin, O. Breast Cancer Chemoresistance: Emerging Importance of Cancer Stem Cells. Surg. Oncol. 2010, 19, 27-32.

(54) Ben-Porath, I.; Thomson, M. W.; Carey, V. J.; Ge, R.; Bell, G. W.; Regev, A.; Weinberg, R. A. An Embryonic Stem Cell-Like Gene Expression Signature in Poorly Differentiated Aggressive Human Tumors. Nat. Genet. 2008, 40, 499-507.

(55) Koch, U.; Lehal, R.; Radtke, F. Stem Cells Living with a Notch. Development 2013, 140, 689-704.

(56) Campos, L. S.; Decker, L.; Taylor, V.; Skarnes, W. Notch, Epidermal Growth Factor Receptor, And Beta1-Integrin Pathways Are Coordinated in Neural Stem Cells. J. Biol. Chem. 2006, 281, 5300-5309.

(57) Brabletz, S.; Bajdak, K.; Meidhof, S.; Burk, U.; Niedermann, G.; Firat, E.; Wellner, U.; Dimmler, A.; Faller, G.; et al. The ZEB1/miR-200 Feedback Loop Controls Notch Signalling in Cancer Cells. EMBO J. 2011, 30, 770-782. 